\title{
Nonlinear Control of Electronic Throttle Based on Backstepping Approach
}

\author{
Junli Wan ${ }^{1, *}$, Quyang Zeng ${ }^{1,2}$, Shiyun Cheng ${ }^{1}$, Xianyong Wu ${ }^{1}$ \\ ${ }^{1}$ College of Electrical Engineering and Renewable Energy, China Three Gorges University, Yichang, China \\ ${ }^{2}$ Three Gorges Vocational College of Electric Power, Yichang, China
}

\author{
Email address: \\ wjlem@163.com (Junli Wan) \\ ${ }^{*}$ Corresponding author
}

\section{To cite this article:}

Junli Wan, Quyang Zeng, Shiyun Cheng, Xianyong Wu. Nonlinear Control of Electronic Throttle Based on Backstepping Approach. American Journal of Electromagnetics and Applications. Vol. 4, No. 1, 2016, pp. 1-7. doi: 10.11648/j.ajea.20160401.11

Received: August 16, 2016; Accepted: September 14, 2016; Published: October 20, 2016

\begin{abstract}
In order to overcome the disadvantage of the non-linear attribute of electronic throttle and the deficiency of traditional PID controller, a control strategy is proposed based on backstepping method, in which the throttle position tracking is taken as control object, and the structure of the electronic throttle is analysed, a mathematical model of the electronic throttle is established. A backstepping controller is also designed based on Lyapunov stability theory. Step, slope and sine waves are taken as target signals respectively to complete tracking control simulation by two control strategies: PID and backstepping, the simulation result indicates that backstepping control has good dynamic characteristics, step response time is less than $100 \mathrm{~ms}$ and its control effect is significantly better than that of PID. In addition, PID simulation can not reflect the torque in actual system reset spring, the simulation is different with actual waveform, and the Backstepping simulation based on the control model of nonlinear system is more in line with the actual, this type of control can effectively solve the contradiction between fast response and large overshoot in time-varying non-linear system.
\end{abstract}

Keywords: Electronic Throttle, Backstepping Control, Position Tracking, Simulate

\section{Introduction}

In recent years, vehicles equipped with electronic throttle control (ETC) [1, 2] systems are gaining popularity, the application of electronic throttle systems has been very popular in the automotive industry [3, 4]. The electronic throttle is essentially a DC-motor-driven valve that regulates air inflow into the vehicle's combustion system. By using the electronic throttle, the engine control unit can correct the throttle position reference value for specific engine operating modes, thus improving vehicle drivability, fuel economy, and emissions $[5,6]$.

The synthesis of a satisfactory ETC system is not easy since the plant is burdened with strong nonlinear effects of stick-slip friction, spring and gear backlash [7]. In order to address this problem, several detailed studies have been performed and control strategies proposed: 1) optimal control techniques, such as model predictive control strategy-based time-optimal control [8], constrained finite time optimal control [9], dynamic programming-based optimal control strategy for both electronic throttle and automatic transmission [10]; 2) linear controller with compensator, such as optimized proportional- integral-derivative (PID) controller with a feedback compensator for friction and limphome effects [11], auto-tuning PID controller with nonlinear friction and limp-home compensators [12]; 3) sliding mode control (SMC) techniques, such as variable structure control with sliding mode [13, 14], neural networks-based sliding mode control [15], etc. Electronic throttle control system is developing toward to the complex control and integration, the use of modern control theory and method which realize intelligent control and improve the adaptive ability of electronic throttle control system are the developing trend of modern automobile.

Because the electronic throttle control system has nonlinear, time-varying, uncertainty [16], it is difficult to achieve higher control precision and better tracking performance by the traditional PID controller [17], to meet fast tracking of the step signal, a large control signal is needed which will cause an 
excessive overshoot and the throttle valve jitter. Considering the PID controller's deficiency, a backstepping control strategy based on Lyapunov stability theory is proposed in this paper, MATLAB/SIMULINK toolbox is used to complete the simulation of electronic throttle control system and achieve a fast and accurate control for optimum opening degree of the throttle. In backstepping control, the nonlinear factors are reflected in controlled quantities directly, which has feedforward compensation effect, so the adjustment time is shorter, hysteresis is not obvious, and the control effect is better.

\section{Electronic Throttke Modeling}

Electronic Throttle is an electromechanical transmission system, which is mainly composed of DC motor, reduction gear, return spring, throttle valve and throttle position sensor, etc. the DC motor is an actuator, which will transmit torque through reduction gear to the throttle shaft to drive the rotation of the throttle valve to control air flow [7], the structure is shown as in Fig 1. Its working principle is: When the driver depresses the accelerator pedal, accelerator pedal position sensor will transmit the driver's information to electronic throttle control unit, from the information obtained, control unit will calculate throttle opening degree using control strategy and output a corresponding control signal to the drive motor, under the effect of the drive motor, the throttle will attain a desired position, during the whole control process, the throttle sensor will feedback continuously the throttle opening degree signals to the control unit, which will continuously compare the target values with the opening degree signals and then make some corrections, until the throttle valve opening degree reaches the target value.

In operation, the throttle valve will be subject to the effect of spring torque applied by the returning spring, the viscous friction and Coulomb friction torques as well as the driving torque provided by the motor. When the motor output torque attain a dynamic equilibrium with the resistance generated by return spring of throttle and friction, throttle opening will keep invariant, the system uses pulse width modulation technology to drive DC motor, to adjust the duty ratio according to position control signal, to change output torque of DC motor, which will realize opening degree control for throttle.

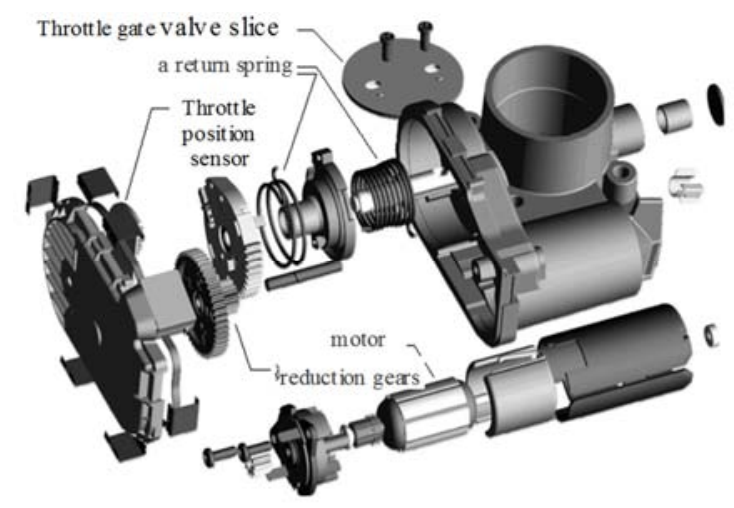

Fig. 1. Structure of the electronic throttle.
A throttle return spring is a torsion spring with a different degree of opening of the throttle valve, the torsional torque applied to the center axis of the throttle valve is nonlinear. Spring torque equation is:

$$
T_{s}=k_{s}\left(\theta-\theta_{0}\right)+D \operatorname{sgn}\left(\theta-\theta_{0}\right)
$$

Where: $k_{s}$ is a torsional spring coefficient, $\mathrm{D}$ is a spring compensation coefficient, $\theta$ is the rotation angle of the throttle valve, $\theta_{0}$ is the equilibrium position of the throttle angle.

In the movement of throttle, a variety of frictions will be produced, the main impact is the viscous friction and Coulomb friction, viscous friction is opposite with the rotation direction of the throttle valve and is proportional to its rotation speed, Coulomb friction is a constant obstruction which is opposite to its speed direction, the total friction torque can be expressed as:

$$
\left\{\begin{array}{l}
T_{d}=k_{d} \omega(t) \\
T_{f}=k_{f} \operatorname{sgn}(\omega(t)) \\
T_{F}=T_{d}+T_{f}=k_{d} \omega(t)+k_{f} \operatorname{sgn}(\omega(t))
\end{array}\right.
$$

Where: $k_{d}$ is a viscous friction coefficient, $k_{f}$ is the Coulomb friction coefficient, $\omega$ is the angular velocity of the throttle.

The torque generated by motor is proportional to the current, which can be expressed as:

$$
T_{m}=k_{m} i(t)
$$

Where $k_{m}$ is the motor torque coefficient.

DC armature circuit current equation is:

$$
L \frac{d i}{d t}+R i(t)=u(t)-k_{v} \omega_{m}(t)
$$

Where $k_{v}$ is the motor counter electromotive coefficient, $R$ is the motor resistance, $L$ is the motor inductance, $\omega_{m}$ is the motor speed, So we have:

$$
\omega_{m}(t)=N \omega(t)
$$

Where is throttle valve speed, $N$ is the gear ratio.

Take motor shaft as a reference point, according to Newton's second law, we have:

$$
J_{m} \frac{d \omega_{m}}{d t}+b_{m} \omega_{m}+T_{L}=T_{m}
$$

Where $J_{m}$ is the moment of inertia of the motor, $b_{m}$ is the damping coefficient of the motor, $T_{L}$ is the motor load.

Consider the impact of nonlinear spring torque and friction, take throttle shaft as a reference point, suppose $J_{g}$ is the moment of inertia of the throttle valve, the throttle 
dynamic equation can be obtained by Newton's second law:

$$
\begin{aligned}
& J_{g} \frac{d \omega}{d t}=N T_{L}-T_{s}-T_{F} \\
& =N\left(k_{m} i-J_{m} \frac{d \omega_{m}}{d t}-b_{m} \omega\right)-k_{s}\left(\theta-\theta_{0}\right)-D \operatorname{sgn}\left(\theta-\theta_{0}\right)-k_{d} \omega_{m}-k_{f} \operatorname{sgn}(\omega)
\end{aligned}
$$

Assume the total moment of inertia $J=J_{g}+N^{2} J_{m}$

From (1) to (7), we obtain the throttle angle dynamic equation as follows:

$$
\left\{\begin{aligned}
\dot{\theta} & =\omega \\
\dot{\omega} & =\frac{N k_{m}}{J} i-\frac{k_{d}+N^{2} b_{m}}{J} \omega-\frac{k_{s}}{J}\left(\theta-\theta_{0}\right) \\
& -\frac{D}{J} \operatorname{sgn}\left(\theta-\theta_{0}\right)-\frac{k_{f}}{J} \operatorname{sgn}(\omega) \\
\dot{i}= & -\frac{R}{L} i-\frac{N k_{v}}{L} \omega+\frac{1}{L} u
\end{aligned}\right.
$$

\section{Backstepping Controoller Design}

The backstepping control principle is using recursive method to design controller [18, 19], some state variables are taken as "virtual control signal". By constructing the Lyapunov function, the final control signal is obtained recursively through a series of "virtual control signal" [20]. It is essentially a kind of hierarchical dynamic error compensation, which is the decomposition of the complex nonlinear system into several subsystems, the order is not exceed the system order in these subsystems, a Lyapunov function is designed in each subsystem, all the derivatives of Lyapunov function in these subsystems must be less than zero to ensure that the system is asymptotically stable [21].

According to the throttle angle dynamic equation (8), let the state variable $x_{1}=\theta, x_{2}=\omega, x_{3}=i$, and let $b_{2}=\frac{N k_{m}}{J}$, $b_{3}=\frac{1}{L}, a_{1}=\frac{k_{d}+N^{2} b_{m}}{J}, a_{2}=\frac{k_{s}}{J}, a_{3}=\frac{D}{J}, a_{4}=\frac{k_{f}}{J}, a_{5}=\frac{R}{L}$, $a_{6}=\frac{N k_{v}}{L}$

Then equation (8) can be simplified as:

$$
\left\{\begin{array}{l}
\dot{x}_{1}=x_{2} \\
\dot{x}_{2}=b_{2} x_{3}+f_{1} \\
\dot{x}_{3}=b_{3} u+f_{2}
\end{array}\right.
$$

Where

$$
\begin{aligned}
& f_{1}=-a_{1} x_{2}-a_{2}\left(x_{1}-\theta_{0}\right)-a_{3} \operatorname{sgn}\left(x_{1}-\theta_{0}\right)-a_{4} \operatorname{sgn}\left(x_{2}\right) \\
& f_{2}=-a_{5} x_{3}-a_{6} x_{2}
\end{aligned}
$$

Define error $e_{1}=x_{d}-x_{1}, x_{d}$ is a reference value, we have:

$$
\dot{\bullet} \dot{e}_{1}=\dot{x_{d}}-\dot{\bullet} \dot{x}_{1}=\dot{x_{d}}-x_{2}
$$

Define virtual control function as:

$$
\lambda_{1}=c_{1} e_{1}+\dot{x}_{d}\left(c_{1}>0\right)
$$

Defined error $e_{2}=\lambda_{1}-x_{2}$

Define Lyapunov function: $V_{1}=\frac{1}{2} e_{1}^{2}$, then:

$$
\dot{V}_{1}=e_{1} \dot{e}_{1}=e_{1}\left(\dot{x_{d}}-x_{2}\right)=e_{1}\left(e_{2}+\dot{x_{d}}-\lambda_{1}\right)
$$

substitute (11) into (12), we obtain:

$$
\dot{V}_{1}=e_{1} e_{1}=-c_{1} e_{1}^{2}+e_{1} e_{2}
$$

when $c_{1}>0$, the error $e_{2}$ tends to zero as $t$ tends to infinity, so $\dot{V}_{1}<0$, therefore, the subsystem is stable from Lyapunov stability theory.

Let $e_{2}=\lambda_{1}-x_{2}$, then $\dot{e}_{2}=\dot{\lambda}_{1}-\dot{x}_{2}=\dot{\lambda}_{1}-b_{2} x_{3}-f_{1}$, define a virtual control function as:

$$
\lambda_{2}=c_{2} e_{2}+e_{1}+\frac{\dot{\lambda}_{1}-f_{1}}{b_{2}}
$$

Defined error $e_{3}=\lambda_{2}-x_{3}$,

Define Lyapunov function: $V_{2}=V_{1}+\frac{1}{2 b_{2}} e_{2}^{2}$, we have:

$$
\begin{gathered}
\dot{V}_{2}=-c_{1} e_{1}^{2}+e_{1} e_{2}+\frac{1}{b_{2}} e_{2} \dot{e}_{2}=-c_{1} e_{1}^{2}+e_{1} e_{2}+\frac{1}{b_{2}} e_{2}\left(\dot{\lambda}_{1}-b_{2} x_{3}-f_{1}\right) \\
=-c_{1} e_{1}^{2}+e_{1} e_{2}+e_{2}\left(e_{3}+\frac{\dot{\lambda}_{1}-f_{1}}{b_{2}}-\lambda_{2}\right)=-c_{1} e_{1}^{2}-c_{2} e_{2}^{2}+e_{2} e_{3}(15)
\end{gathered}
$$

Similarly, when $t \rightarrow \infty, e_{3} \rightarrow 0$, then $\dot{V}_{2}<0$. Subsystem is stable.

Define the Lyapunov function: $V_{3}=V_{2}+\frac{1}{2 b_{3}} e_{3}^{2}$, then,

$$
\begin{gathered}
\dot{V}_{3}=\dot{V}_{2}+\frac{1}{b_{3}} e_{3} \dot{e}_{3}=-c_{1} e_{1}^{2}-c_{2} e_{2}^{2}+e_{2} e_{3}+\frac{1}{b_{3}} e_{3}\left(\dot{\lambda}_{2}-b_{3} u-f_{2}\right) \\
=-c_{1} e_{1}^{2}-c_{2} e_{2}{ }^{2}+e_{2} e_{3}+e_{3}\left(\frac{\dot{\lambda}_{2}-f_{2}}{b_{3}}-u\right)
\end{gathered}
$$

Let $u=c_{3} e_{3}+e_{2}+\frac{\dot{\lambda}_{2}-f_{2}}{b_{3}},\left(c_{3}>0\right)$ and substitute it into (16), we obtain: 


$$
\dot{V}_{3}=-c_{1} e_{1}^{2}-c_{2} e_{2}^{2}-c_{3} e_{3}^{2}<0
$$

It can be seen: $V_{3}$ is asymptotically stable, then $e_{1}, e_{2}, e_{3}$ are asymptotically stable, which will ensure global stability asymptotically of the system. Then the last control signal can be expressed as:

$$
u=c_{3} e_{3}+e_{2}+\frac{\dot{\lambda}_{2}-f_{2}}{b_{3}}
$$

The formula (17) shows that the linear part of the control law and the PID algorithm are Similarity, and the calculation is not complicated, easy to implement the engineering controller.

\section{Simulation and Experimental Results}

The target signals are: a step signal, a ramp signal and a sine signal, simulation results with two control methods are shown as in Fig. 2 and Fig. 3

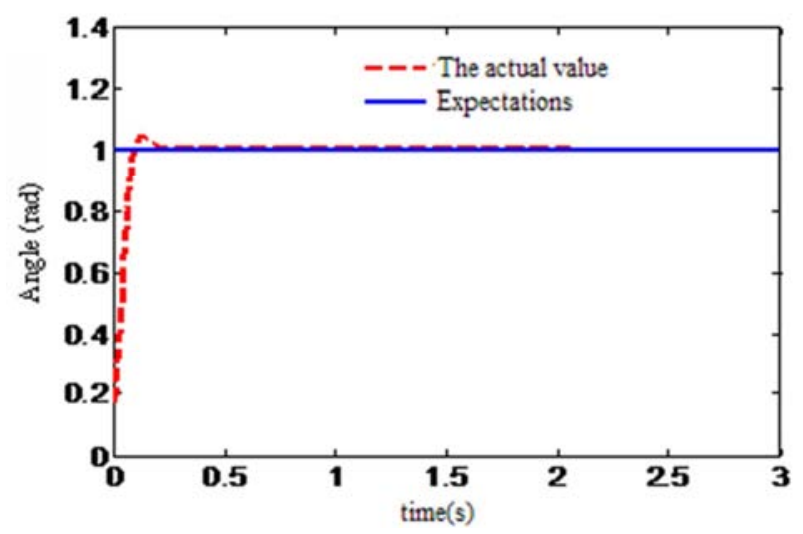

a

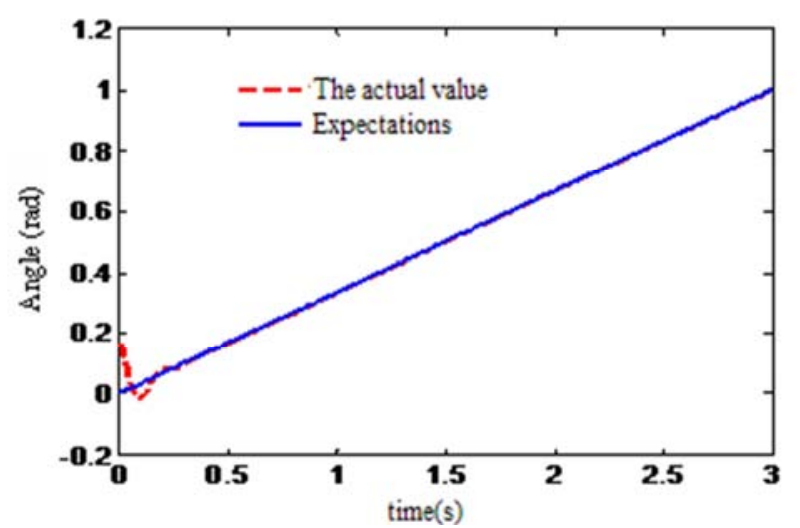

b

\subsection{Simulation of PID and Backstepping Control Strategies}

To compare the effects of different control strategies for electronic throttle control, two control strategies of PID and backstepping are simulated in this paper. Electronic throttle parameters are not required for conventional PID control, only proportional, differential and integral coefficients of the control signal are needed to be determined to make the tracking error reach the set value. But the backstepping control build its simulation model for position tracking control by throttle parameters and angle dynamic equation, and derive a stable control law by constructing Lyapunov function [22].

To make the design of backstepping controller be applied to actual system, a controller module is built by $\mathrm{S}$ function in MATLAB. The input signal is DC motor control voltage $u$, and the output is the position of the throttle opening. The parameter are taken as follows:

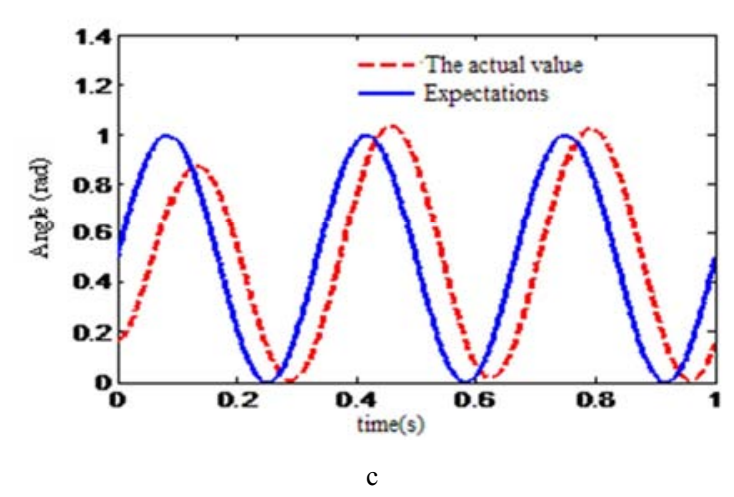

Fig. 2. Simulation curve of PID control.

a PID step signal tracking

b PID ramp signal tracking

c PID sine signal tracking

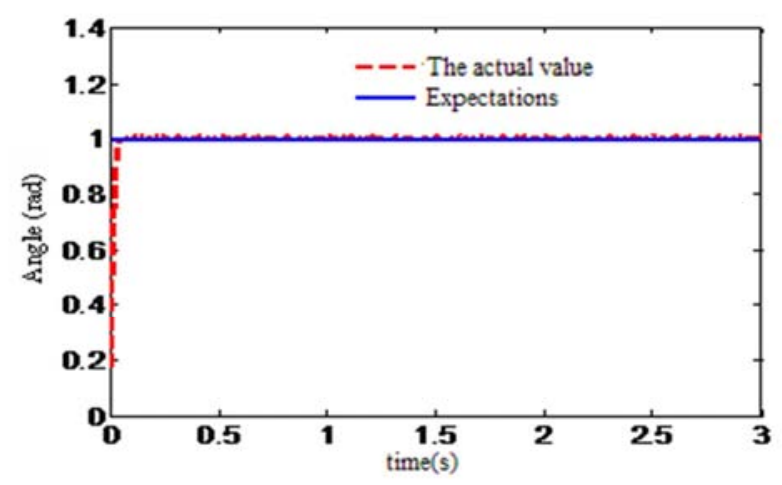




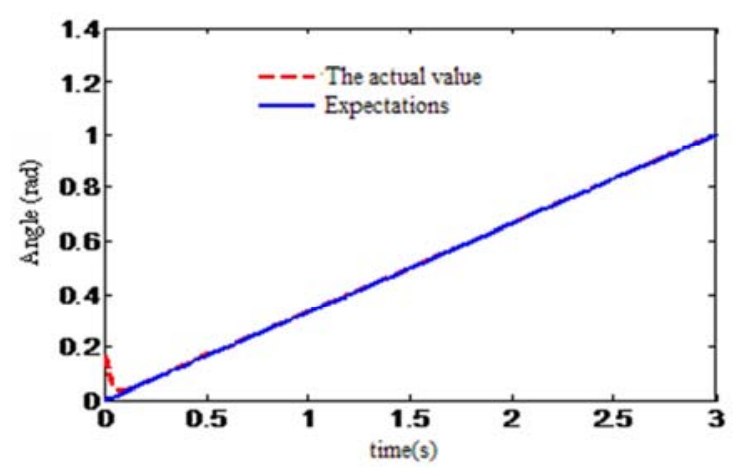

b

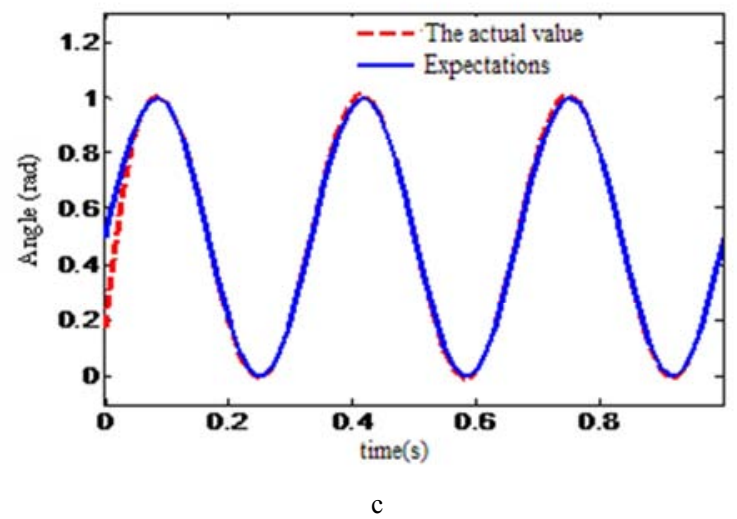

Fig. 3. Simulation curve of backstepping control.

a Backstepping control step signal tracking

b Backstepping control ramp signal tracking

c Backstepping control sine signal tracking

\subsection{The Simulation Result Analysis}

- As can be seen from Fig. 2: throttle opening is obviously overshoot for a given target step signal when PID control is used, overshoot is about $5 \%$, when the steady-state error is $1 \%$, the response time is about $280 \mathrm{~ms}$. No overshoot is occurred when backstepping control is used, response time is within $100 \mathrm{~ms}$, the steady-state error is less than $1 \%$.

- Fig. 2, Fig. 3 shows that: when tracking the ramp signal, the response time of backstepping control is half of the time of PID control; tracking the sine wave signal in PID control with a significant lag, and the error is large, the use of backstepping control has no obvious hysteresis and the error is minimal.

- Traditional PID control only take error as a basic control variable, and the proportional coefficient, integral coefficient and differential coefficient keep unchanged in adjustment process, so the control effect for nonlinear systems is not ideal. But in backstepping control, the nonlinear factors are reflected in controlled quantities directly, which has feed-forward compensation effect, so the adjustment time is shorter, hysteresis is not obvious, and the control effect is better.

\subsection{Electronic Throttle Control System Experiments and Discussion}

Fig. 4 is a block diagram of the control system's hardware, and the system is mainly composed of a single-chip microcontroller control module, a sensor module, an AD conversion module, a driving module and a display module.

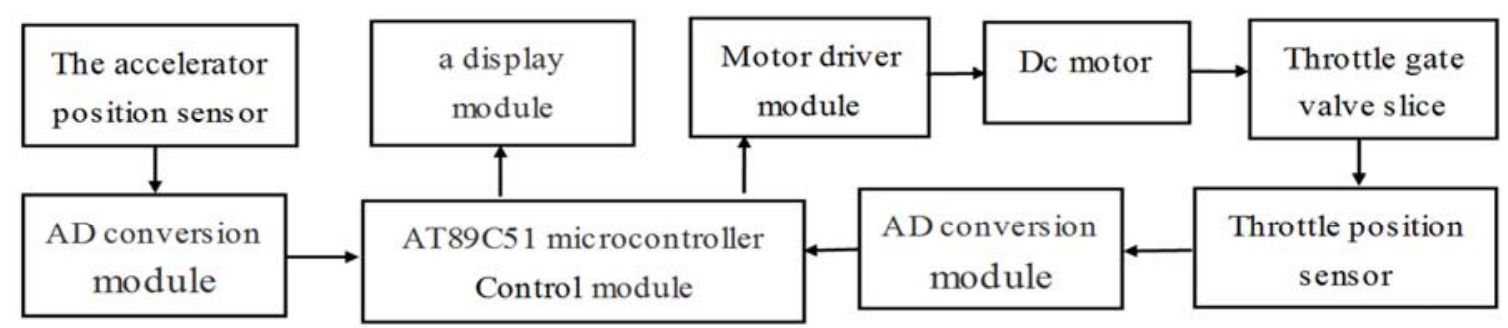

Fig. 4. The block diagram of the control system's hardware.

Electronic Throttle Control System is shown as in Fig 5. The BOSCH DV-E5 throttle gate with DC motor and position sensor is used in experiment, an intelligent power chip TLE6209R with only one PWM signal and direction signal is chosen as the drive for DC motor, a closed loop control system is composed of ATmegal6 single-chip computer, which realize data acquisition and processing for accelerator pedal and throttle valve position and PWM output. The PID control algorithm is used in experiment, the parameters of PID controller are: proportional coefficient $K_{p}$ $=0.5865$, integral coefficient $K_{i}=0.2346$, differential coefficient $K_{d}=0.0235$. The waveforms which the feedback values track the target values are shown in Fig. 6.

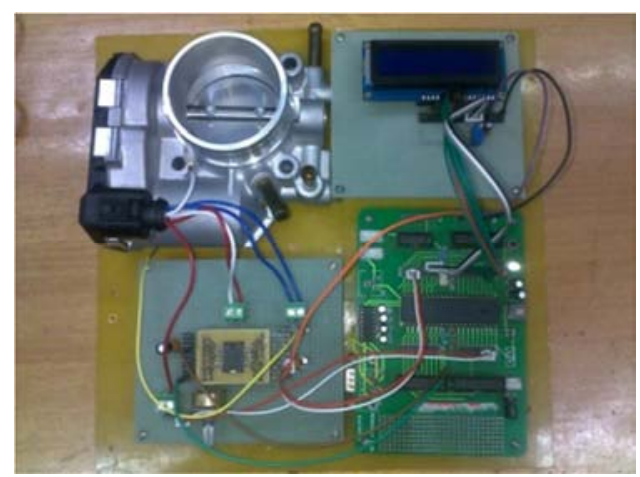

Fig. 5. Electronic Throttle Control System. 


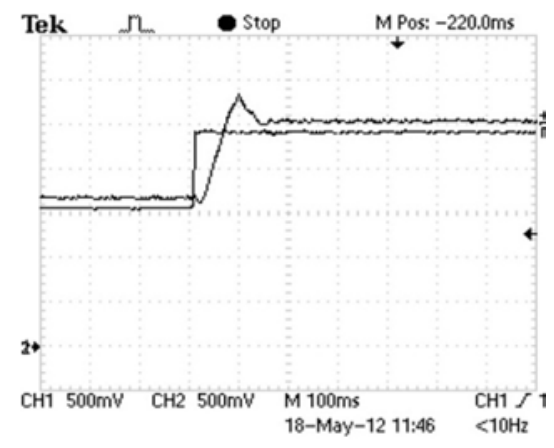

a

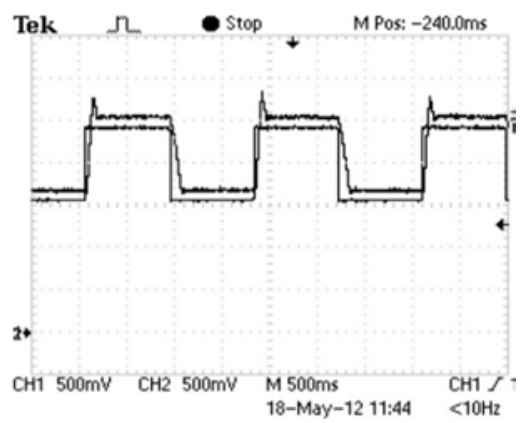

b
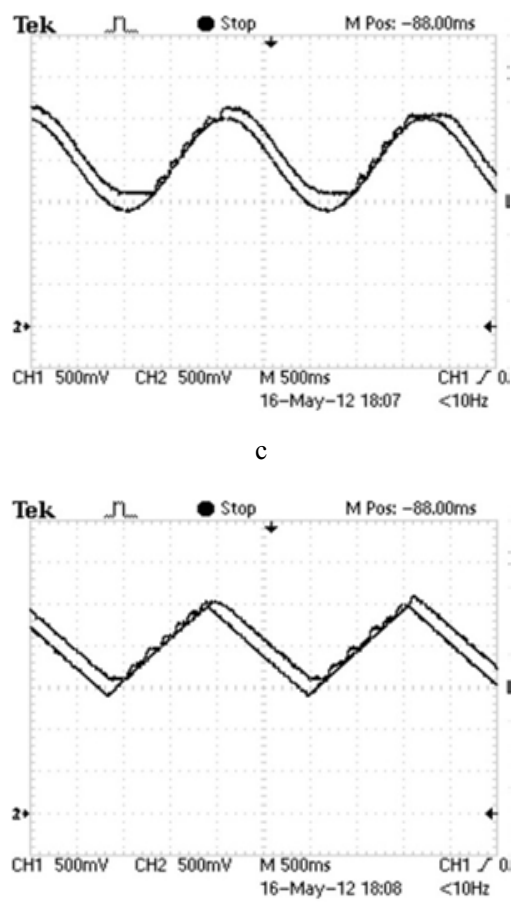

d

Fig. 6. Four groups of different waveform tracking test.

a step signal tracking

b square wave signal tracking

c sine signal tracking

d triangular wave signal tracking

Fig. 6 (a) shows that when step input is applied, the overshoot is $27 \%$, the adjustment time is $280 \mathrm{~ms}$, the reason the overshoot is so high is that a larger control driving signal is needed to ensure fast tracking.
When sine and triangular waves are applied, Fig. 6 (c) and Fig. 6 (d) show that: In rising stage of target value, the tracking accuracy is low and there is significant fluctuation, in the decline stage of target value, the tracking effect is better. It also shows the actual system must overcome the spring torque exerted by the return spring during the control of opening increase of throttle valve, and a return spring torque help to realize tracking control when opening decreases. Since the PID method is not based on system model, the simulation result is naturally not influenced by the spring torque, so there are differences between PID simulation and the actual result, the backstepping control method includes the system nonlinear factors, therefore, it has the advantages of nonlinear control.

\section{Conclusion}

In this paper, a mathematical model of electronic throttle is built, a backstepping controller is designed based on Lyapunov stability theory, step, ramp and sine-wave signals are used as target signals for simulation, two simulation curves under backstepping and PID control are analyzed and compared, simulation results show that backstepping control has good dynamic features, which is better than that of PID. Of course, it is easier using PID control in the actual control system, but it is difficult to solve the contradiction between fast response and large overshoot, which will lead to electronic throttle valve jitter, but backstepping control provides a new method to effectively solve this kind of nonlinear control. With the rapid development of computer technology and digital electronics, backstepping controller will be widely used in practical nonlinear control systems.

\section{Acknowledgments}

This work was supported by the National Natural Science Foundation, China (No. 61074091) and the Natural Science Foundation of Hubei Province, China (No. 2010CDB10803 ).

\section{References}

[1] Yuan, X. F., Wang, Y. N. "A novel electronic throttle valve controller based on approximate model method," IEEE Trans. Ind. Electron. 2009, 56 (3), pp. 883-890.

[2] H. Yun-feng, L. Qi-fang, S. Peng-yuan, et al. "Design of an ADRC-based electronic throttle controller," Proc. of the 30th Chinese Control Conference, Yantai, China, 2011, pp. 63406344 .

[3] Y. Xiao-fang, W. Yao-nan, W. Liang-hong, et al. "Neural Network Based Self-Learning Control Strategy for Electronic Throttle Valve," IEEE Transactions on Vehicular Technology, 2010, 59 (8), pp. 3357-3765.

[4] Yuan X., Wang Y., Sun W., et al. "RBF Networks-Based Adaptive Inverse Model Control System for Electronic Throttle," IEEE TRANSACTIONS ON CONTROL SYSTEMS TECHNOLOGY, 2010,18 (3), pp.750-756. 
[5] Yuan, X., Li, S., Wang,Y., et al. "LianghongWu Parameter identification of electronic throttle using a hybrid optimization algorithm," Nonlinear Dyn, 2011, 63: pp. 549-557.

[6] GENG J F, JIAO Y Y, LIU Z Y. "Fuzzy control on the electronic throttle," Chinese Journal of Scientific Instrument, 2006, 27 (6): pp.847-848.

[7] YU H Y, LU Y J, YANG S C. "Research on Electronic Throttle Control System Based on Position Feedback," Automobile Technology, 2007, (8), pp.8-11.

[8] M. Vasak, M. Baotic, I. Petrovic, and N. Peric, "Hybrid theory-based time-optimal control of an electronic throttle," IEEE Trans. Ind. Electron. 2007, 54 (3), pp. 1483-1494.

[9] M.Vasak, M. Baotic, M. Morari, I., et al. "Constrained optimal control of an electronic throttle," Int. J. Control, 2006,79 (5), pp. 465-478.

[10] D. Kim, H. Peng, S. Bai, et al. "Control of integrated powertrain with electronic throttle and automatic transmission," IEEE Trans. Control Syst. Technol. 2007,15 (3), pp. 474-482.

[11] J. Deur, D. Pavkovic, N. Peric, et al. "An electronic throttle control strategy including compensation of friction and limphome effects," IEEE Trans. Ind. Appl. 2004, 40 (3), pp. 821-834.

[12] D. Pavkovic, J. Deur, M. Jansz, et al. "Adaptive control of automotive electronic throttle," Control Eng. Pract. 2006,14 (2), pp. 121-136.

[13] Y. Pan, U. Ozguner, and O. H. Dagci, "Variable-structure control of electronic throttle valve," IEEE Trans. Ind. Electron. 2008, 55 (11), pp. 3899-3907.

[14] K. Nakano, U. Sawut, K. Higuchi, et al. "Modelling and

\section{Biography}

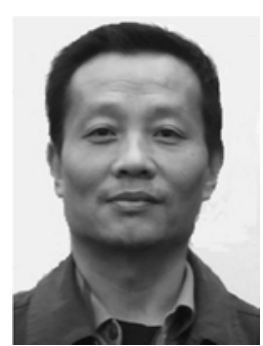

Junli Wan (Communication author) was born in 1957. Now he is a professor and Master supervisor in the College of Electrical Engineering \& Renewable Energy, China Three Gorges University. His present research interests include intelligent instrument and intelligent control, as well as related theory and technique.

Address: College of Electrical Engineering and Renewable Energy, China Three Gorges University, No.8 Daxue Avenue, Yichang 443002, Hubei Province, China

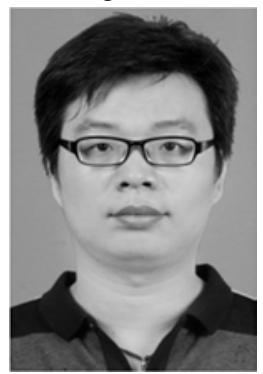

Shirun Cheng was born in 1989. Now he is a student in the College of Electrical Engineering \& Renewable Energy, China Three Gorges University. He present research interests are about the smart grid and intelligent control.

Address: College of Electrical Engineering and Renewable Energy, China Three Gorges University, No.8 Daxue Avenue, Yichang 443002, Hubei Province, China observer- based sliding-mode control of electronic throttle systems," ECTI Trans. Electr. Eng. Electron. Commun. 2006, 4 (1), pp. 22-28.

[15] M. Baric, I. Petrovic, and N. Peric, "Neural network-based sliding mode control of electronic throttle," Eng. Appl. Artif. Intell. 18, no. 2005,8, pp. 951-961.

[16] SUN W, ZHOU Y H, XI M L. "Method for parameter optimization of nonlinear PID controller," Computer Engineering and Applications, 2010, 46 (28), pp. 244-248.

[17] ZENG, Q. Y, WAN, J. L. "Nonlinear PID control of electronic throttle valve," Proceeding of the 2nd International Conference on Electrical and Control Engineering, Yichang, China, IEEE, 2011,pp.722-724.

[18] Sartori, D., Quagliotti, F., Rutherford, M.J., et al. "Design and Development of a Backstepping Controller Autopilot for Fixed-wing UAVs," Denver University Unmanned Systems Research Institute, CO, USA, Tech. Rep. DU2SRI-2013-12001 (2013).

[19] LIU J K, SUN F C. "Nominal model-based sliding mode control with backstepping for 3-axis flight table," Chinese Journal of Aeronautics, 2006, 19 (1), pp. 65-71.

[20] DONG W H, SUN X X, LIN Y, "Adaptive backstepping control: development and applications," Control and Decision, 2006, 21 (8), pp.1081-1086.

[21] Yao, J., Jiao, Z., Yao, B. et al. "Nonlinear adaptive robust control of electro hydraulic load simulator," China J. Aeronaut. 2012, 25 (5), pp. 766-775.

[22] W. Sun, H. Gao, and O. Kaynak, "Adaptive backstepping control for active suspension systems with hard constraints," IEEE/ASME Trans. Mechatronics, 2013, 18 (3), pp. 10721079.

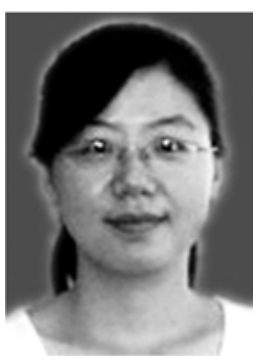

Quyang Zeng was born in 1983, received bachelor degree from Wuhan University of Science and Technology in 2005, and received Master degree from China Three Gorges University in 2012. Now she is a lecturer in Gorges Vocational College of Electric Power. Her present research interests include motor driving and nonlinear control system.

Address: Three Gorges Vocational College of Electric Power, Yichang 443000, China,

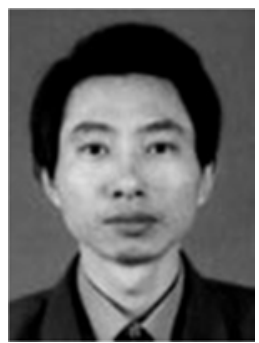

Xianyong Wu was born in 1963. Now he is a professor and Master supervisor in the College of Electrical Engineering \& Renewable Energy, China Three Gorges University. His present research interests include nonlinear control, complex network. Address: College of Electrical Engineering and Renewable Energy, China Three Gorges University, No.8 Daxue Avenue,

Yichang 443002, Hubei Province, China 